\title{
Der «Schweizer Eid»
}

Bernhard Egger, Ruth Baumann-Hölzle, Max Giger, Claudia Käch, Audrey Kovatsch, Diana Meier-Allmendinger, Judit Pòk Lundqvist, Pascal Schai, Jean-Pierre Wils

\author{
«Manche Dinge werden beschädigt oder herabgesetzt, \\ wenn man sie in Waren verwandelt.»
}

Michael J. Sandel, Was man für Geld nicht kaufen kann

\section{Zur Diagnose}

In modernen, hochkomplexen Gesellschaften sind Institutionen einem permanenten Wandel ausgesetzt. Die Gesundheitsinstitutionen bilden da keine Ausnahme, im Gegenteil. Die politisch induzierten Reformvorhaben wechseln regelmässig. Marktanforderungen sind allgegenwärtig und mit ihnen wächst die Erwartung, Gewinnmaximierung bei gleichzeitiger Kostenersparnis sei auch in den Gesundheitsinstitutionen ein wichtiges Ziel. Der wirtschaftliche Wettbewerb nötigt zu Rationalisierungsmassnahmen, zur Straffung der Abläufe und zur fortwährenden Suche nach Effizienzsteigerungen und nach Massnahmen zur Erhöhung der Kosteneffektivität. Krankenhäuser haben vielerorts ein betriebliches Aussehen bekommen. Wirtschaftssoziologisch gut erforscht ist eine Entwicklung, die man unter dem Stichwort Ökonomisierung zusammenfassen kann: Während die Aufforderung, sich kostenbewusst zu verhalten, zunächst die Vermeidung von Verlusten bedeutet, müssen die Akteure sich im späteren Verlauf der Soll-Erwartung der Gewinnsteigerung unterwerfen, so dass die Erzielung von Gewinnen zum wichtigsten Ziel der Organisation wird. Aus betriebswirtschaftlicher Perspektive lassen sich

\section{Le «Serment Suisse»}

Il y a trois ans, une commission interprofessionnelle composée de médecins et de représentants de l'économie, de l'éthique, de la philosophie et d'étudiants en médecine s'est formée au sein de l'institut Dialog Ethik à Zurich. Elle s'était fixée pour mission de réfléchir à un nouveau serment, un nouvel engagement des médecins, de le formuler et de rechercher des possibilités de mise en œuvre. Dans l'intervalle, ce projet a bien avancé: l'attention des médias en Suisse et à l'étranger était et reste très soutenue. Des discussions animées ont eu lieu dans les organisations spécialisées de Suisse. En juin, après l'assemblée plénière de la fmCH, l'assemblée des délégués de la FMH a à son tour décidé de soutenir la diffusion du nouveau serment. gegen diese Zielvorgabe kaum Einwände erheben. Ob und in welchem Masse sie generell und im Besonderen in medizinischen Institutionen sinnvoll ist, dürfte dagegen fraglich sein. In Spitälern begegnen sich nämlich zwei Systeme - das notwendigerweise kalkulierende, «kalte» System der Ökonomie und das dem Patientenwohl verpflichtete, «warme» System ärztlichen, aber auch pflegerischen und therapeutischen Handelns. Quantitatives, wirtschaftliches Denken einerseits und die professionelle Autonomie andererseits, die wesentlich mit der Kunstfertigkeit ärztlichen Handelns zusammenhängt, müssen hier balanciert werden. $\mathrm{Zu}$ dieser Balance gehört die Einsicht, dass gute Medizin auf eine stabile ökonomische Basis angewiesen ist. Auch fehlendes Kostenbewusstsein und mangelhaft ausgeprägte wirtschaftliche Sensibilität unterminieren angemessenes ärztliches Handeln.

Aber Ökonomisierung meint etwas anderes: die Unterordnung der medizinischen Kunstfertigkeit unter die Direktive der Ökonomie. Die Einschränkung der Autonomie ärztlichen Handelns bildet die unmittelbare Folge dieser Entwicklung. Während einst die Ökonomie subsidiär der Medizin zugeordnet war, hat sich dieses Verhältnis nun umgekehrt: Ärztliches Handeln steht im Dienste der betriebswirtschaftlichen Ausrichtung der Institution. Medizinischer Erfolg meint in diesem Falle ökonomischer Erfolg. Inzwischen sind die Konsequenzen dieser Umwandlung äusserst gut dokumentiert. Es ist geradezu die Rede von einer «De-Professionalisierung», von einem Verlust des typischen Profils ärztlichen Handelns. Berufsbild und Berufsethos sind in Mitleidenschaft gezogen. Standesorganisationen und ethische Institutionen weisen seit Jahren besorgt auf diese Transformation des Berufs hin. Wenige Beispiele müssen an dieser Stelle genügen.

\section{Hinweise}

Eine Arbeitsgruppe der SAMW wies bereits im Jahre 2007 auf «die Spannungen zwischen dem auf den Menschen und auf das Menschliche zentrierten Auftrag des Gesundheitswesens und den immer mehr dominierenden ökonomischen Faktoren» hin [1], Spannungen, die «ein beträchtliches Konfliktpotenzial» enthalten. In einem Positionspapier der SAMW mit dem Titel 
«Medizin und Ökonomie - wie weiter?» aus dem Jahre 2014 [2] findet sich folgende, zutreffende Beobachtung: Es habe sich «die Position der Ökonomie in der Hierarchie der Gesundheitseinrichtungen gewandelt, von einer auf die Verwaltung beschränkten, eher buchhalterischen Funktion hin zum Management und der Exekutive, die die strategischen Entscheidungen trifft. [...] Wichtig ist, dass die professionelle Integrität und Autonomie medizinischer Fachpersonen erhalten bleibt. [...] Das bedeutet nicht, ohne Absprachen frei agieren zu können, aber es muss die Möglichkeit erhalten bleiben, als 'Anwalt' der Patienteninteressen aufzutreten und sich primär für das Wohl der Patienten zuständig zu fühlen.»

Und Anfang April 2016 veröffentlichte der Deutsche Ethikrat eine überaus wichtige und ausgewogene Stellungnahme mit dem Titel «Patientenwohl als ethischer Massstab für das Krankenhaus» [3]. Zu den Gefährdungen jenes Patientenwohls zählt das Dokument den Konflikt zwischen dem «jeweiligen Berufsethos» und der «Berufswirklichkeit» der im Krankenhaus ärztlich und pflegerisch Tätigen. In diesem Zusammenhang ist davon die Rede, dass die ökonomischen Rahmenbedingungen, die zu zunehmendem Arbeitsdruck und wachsender Arbeitsverdichtung führen, mit dem beruflichen Selbstverständnis in Konflikt geraten. Zwei «Normsysteme» prallen aufeinander-das des Patientenwohls (und somit das des medizinischen Berufsethos) und die «Primärorientierung» (S. 70) an ökonomischen, marktförmigen Kriterien. Die Formulierung "Primärorientierung» signalisiert das Problem einer Vorzugsordnung, denn die ökonomische Bilanzierung medizinischen Handelns sind diesem Handeln keineswegs prinzipiell abträglich. Über die Rangordnung dieser Normsysteme muss allerdings Klarheit gewonnen werden - zugunsten des medizinethisch erforderlichen Primats des Patientenwohls.

Auf dem Spiel steht nichts weniger als das Selbstverständnis des ärztlichen Berufs. Die Ehre oder Würde dieses Berufs, die bereits im (inzwischen in etlichen Teilen veralteten) Eid des Hippokrates zum Ausdruck kam, hatten im Wesentlichen mit der Unabhängigkeit (Autonomie) und der Kunstfertigkeit der Profession zu tun. Unabhängigkeit meint, dass der Massstab ärztlichen Urteilens und Handelns medizinischer Natur ist. Kunstfertigkeit meint, dass die Praktiken von Ärzten und Ärztinnen nicht nur auf angewandtem Wissen und technischem Können beruhen, weshalb sie kein standardisiertes Produkt auf den Markt bringen, sondern das Ergebnis fallgerechten, auf den einzelnen Patienten bezogenen Entscheidens sind. Aus diesem Grund sind Patienten und Patientinnen letzten Endes keine Kunden.

\section{Eine unaufhaltsame Entwicklung?}

Nicht selten wird die Auffassung vertreten, die oben, in unserer "Diagnose», umrissene Transformation sei unaufhaltsam. Die dort beschriebene Ökonomisierung und die mit ihr zusammenhängende De-Professionalisierung seien nun einmal Kennzeichen einer modernen Gesellschaft, und die Klagen, wie sie in den «Hinweisen» soeben zu vernehmen waren, müssten als Ausdruck mangelnder Anpassungsbereitschaft interpretiert werden. Aber ist das tatsächlich der Fall? In diesem Zusammenhang lohnt es sich, die Aufmerksamkeit kurz auf eine Diagnose des Soziologen Niklas Luhmann zu richten. In modernen Gesellschaften, so schreibt Luhmann in einem wichtigen Beitrag zum Gesundheitswesen [4], habe sich «ein System der Geldwirtschaft ausdifferenziert und hat gesellschaftlich fundierte Institutionen des Helfens verdrängt. Geld ist das effektivere funktionale Äquivalent für Hilfe und Dankbarkeit.» (S. 180) Dieser Prozess sei modernitätstypisch: Unterschiedliche Berufe, die einst als gesonderte Berufsstände mit eigenem Ethos existierten, seien gleichsam homogenisiert worden, indem Leistungen immer mehr wettbewerblich organisiert und ihren Wert in finanziellen Grössenordnungen abgebildet wurden Zweifelsohne habe dieser Prozess zu enormen Steigerungen in Produktion und Effizienz geführt.

\section{Während einst die Ökonomie subsidiär der Medizin zugeordnet war, hat sich dieses Verhältnis nun umgekehrt.}

Zu einer Fundamentalkritik gibt es also keinen Anlass. Aber die angedeutete Homogenisierung hat gleichzeitig zu einer Aushöhlung, also zu einem langsamen Verschwinden berufstypischer Standards geführt. Bestimmte Berufszweige sind von dieser Transformation besonders betroffen - der Arztberuf, die pflegerischen und therapeutischen Berufe, der Beruf des Hochschullehrers. Vor allem die betriebsförmige Reorganisation dieser Berufsbilder führt zu einer Aushöhlung ihres traditionellen Selbstverständnisses. In diesem Zusammenhang droht Wertvolles und Einzigartiges verloren zu gehen.

«Professionen haben sich gebildet zur Hilfe bei ungewöhnlichen Lagen, vor allem Lebensrisiken, angesichts von Angst, Tod, nicht eindämmbarem Streit. Sie beschaffen Sicherheit und Problemlösungen durch spezialisierte Techniken des Umgangs mit solchen Problemen; ferner durch eine auf Helfen ausgerichtete Berufsmoral und durch hohes Sozialprestige, das aus den Notlagen des Lebens heraushebt und situationsmässige Überlegenheit, Dispositionsfreiheit und Unan- 
Der Text des «Schweizer Eids» ist auf Deutsch und Englisch abrufbar unter www.saez.ch $\rightarrow$ Aktuelle Ausgabe oder Archiv $\rightarrow$ 2017 $\rightarrow$ Ausgabe 2017/40. Die französische und italienische Fassung sind in Vorbereitung

La version française est en préparation.

La versione italiana è in

preparazione.

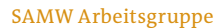
Berufsbilder» (2007) Die zukünftigen Berufs bilder von Ärztinnen/ Ärzten und Pflegenden in der ambulanten und klinischen Praxis. Schweizerische ÄrzteSchweizerische Ârzte1942-52.

Swiss Academies Com munications, Vol. 9, No 4, 2014, 32 .

http://www ethikrat. org/dateien/pdf/steorg/dateien/pdf/stelungnahme-patiente wohl-als-ethischermassstab-fuer-daskrankenhaus.pdf

Luhmann, Niklas: Formen des Helfens im Wandel der gesellschaftWandel der gesellschaftlichen Systeme, in: Soziologische Aufklärung 2, Wiesbaden 2005 , 167-86.

Korrespondenz: Dr. theol. Ruth BaumannHölzle

Institut Dialog Ethik Schaffhauserstrasse 418 CH-8050 Zürich info[at]dialog-ethik.ch greifbarkeit sichert. Zu all dem gehört die Prätention, dass die Hilfe nicht im eigenen Interesse des Helfenden liegt und daher nicht reziprok vergolten, sondern nur 'honoriert' wird. Diese für die klassischen Professionen eigentümliche Kombination von Problembezug, Freiheiten und Bindungen gehört in hochkultivierten Gesellschaften und wird heute nur noch als Attrappe fortgeführt.» (Luhmann 2005, S. 174)

Nun sollte man sich vor Romantisierung hüten. Diese soziologische These behauptet keineswegs, dass der Altruismus in vormodernen Gesellschaften im medizinischen Alltag faktisch dominierte. Es gibt keinen Grund, das zu vermuten. Aber das ärztliche Berufsbild war geprägt von einem «Ethos», in dessen Mittelpunkt die Autonomie der medizinischen Tätigkeit und die verbindliche Ausrichtung am Patientenwohl standen. Und es gibt wiederum gute Gründe, diese Normen zu verteidigen. Denn der Prozess der Ökonomisierung ist weder unumkehrbar noch eine Grösse, der wir machtlos gegenüberstehen. Die Dominanz der Ökonomie ist ein gewachsenes Modell, das politischer Herkunft ist und nicht wie ein Naturgesetz über unsere Gesellschaften gekommen ist. Deshalb sind Auswege aus diesem Modell möglich und mit Sicherheit dort notwendig, wo es einen Schaden anrichtet. Das ist im Gesundheitswesen der Fall.

\section{Ein neuer Eid für Ärzte und Ärztinnen}

Wer unsere Diagnose teilt - die Feststellung eines wachsenden Konflikts zwischen dem berufseigenen Ethos und der zunehmenden Ökonomisierung der ärztlichen Praxis mit ihren de-professionalisierenden Effekten -, wird die Augen vor der Notwendigkeit eines neuen Eides nicht verschliessen können. Ein Eid hat heute mit der Wahrung von Standesprivilegien nichts mehr zu tun. Er bringt vielmehr die durchaus stolze Berufsmoral und die Würde einer Profession zum Ausdruck, indem er Haltungen oder Tugenden charakterisiert, die mit dem Beruf des Arztes intrinsisch verbunden sind und von Aussenstehenden mit dieser Berufsgruppe zu Recht assoziiert werden. Ein Eid soll das Berufsethos gegen Erwartungen ökonomischer und politischer Natur verteidigen, die den moralischen Prinzipien eines Berufs widersprechen. Indem ein Eid auch tatsächlich geleistet wird, realisiert er die Selbstverpflichtung eines Mitgliedes der Berufsgruppe auf jene Prinzipien. Weil ein Eid oder ein Gelöbnis Teil einer organisatorisch verfassten Profession ist, geniesst die Person, die unter Eid steht, den Schutz dieser Organisation bzw. die Solidarität ihrer Mitglieder. Dar- über hinaus bildet der Eid das Vertrauen ab, das Ärzte und Ärztinnen zueinander haben müssen und das die Patienten und Patientinnen zu ihnen haben dürfen. Und die eidesstattlich verfasste Organisation stellt ein mächtiges Bollwerk gegen berufsfremde Übergriffe dar. Moral ist keineswegs wirkungslos.

Eide galten lange Zeit als Relikte aus vergangenen Zeiten. Ausnahmen bildeten Eide auf die Verfassung oder solche, die im Zeugenstand abgelegt werden mussten. Ansonsten standen sie im Verdacht, Standesprivilegien zu verteidigen. Um den «Eid des Hippokrates» war es still geworden. Auch das «Genfer Ärztegelöbnis»

Der Prozess der Ökonomisierung ist weder unumkehrbar noch eine Grösse, der wir machtlos gegenüberstehen.

(1948/1983) und die beiden "Deklarationen des Weltärztebundes» (Helsinki 1964, Tokio 1975) führten ein Dasein im Unauffälligen. Mittlerweile kann jedoch von einer regelrechten Renaissance der Eide gesprochen werden. In der Finanzwelt wird offen über Eide debattiert und werden diese auch implementiert. In der Bundesärztekammer Deutschlands herrscht die Auffassung vor, dass «im Rahmen einer Feierstunde» ein ärztliches Gelöbnis der «Profanisierung» und «Trivialisierung» des Berufsbildes entgegenwirken müsse. Die Stiftung des "American Board of Internal Medicine» hat zusam men mit anderen US-amerikanischen medizinischen Föderationen bereits im Jahre 2002 eine "Charta» verfasst, die auf die Prinzipien des ärztlichen Berufsethos aufmerksam machte. An zahlreichen medizinischen Fakultäten verlangen die Studierenden nach der Ablegung eines Eides bzw. eines Gelöbnisses. Allerdings ist ein zeitgemässes Gelöbnis bislang eher im Vagen geblieben. Oft wird über die Notwendigkeit eines Gelöbnisses «im Sinne» des hippokratischen Eides gesprochen. Ein neuer Eidtext fehlte bislang. Mit dem Schweizer Eid liegt nun endlich ein zeitgemässer Eidtext* vor.

Ein solches Gelöbnis kann und soll eine Standesordnung nicht ersetzen. Aber es bildet gleichsam deren Fundament. Im Bilde gesprochen: Der Eid oder das Gelöbnis kann als Verfassungstext, als tugendethische Verfassung betrachtet werden, auf die sich die Ärzte und Ärztinnen feierlich verpflichten. Sie wollen die dort formulierten Haltungen zur Grundlage ihres Handelns machen und bekennen sich öffentlich dazu. Standesordnungen enthalten ihrerseits sozusagen die Gesetze, die aus dieser Verfassung hervorgehen und daraufhin zu prüfen sind, ob sie mit ihr übereinstimmen. Die Zeit für einen neuen Eid ist reif. 